\title{
Review Article \\ Contemporary Review of the Management of Brain Metastasis with Radiation
}

\author{
Deepak Khuntia \\ Varian Medical Systems, 3100 Hansen Way, E-175, Palo Alto, CA 94340, USA \\ Correspondence should be addressed to Deepak Khuntia; deepak.khuntia@varian.com
}

Received 18 August 2014; Accepted 16 February 2015

Academic Editor: Aimee J. Nelson

Copyright (C) 2015 Deepak Khuntia. This is an open access article distributed under the Creative Commons Attribution License, which permits unrestricted use, distribution, and reproduction in any medium, provided the original work is properly cited.

Brain metastases are an important cause of morbidity and mortality, afflicting approximately 200,000 Americans annually. The prognosis for these patients is poor, with median survivals typically measured in months. In this review article, we present the standard treatment approaches with whole brain radiation and as well as novel approaches in the prevention of neurocognitive deficits.

\section{Introduction}

Brain metastases (BrM) represent an important cause of morbidity and mortality and are the most common intracranial tumors in adults, occurring in up to $30 \%$ of adult cancer patients $[1,2]$. It is estimated that up to 170,000 new cases of brain metastases occur in the United States each year $[3,4]$. It is speculated that the annual incidence is rising for several reasons, including an aging population, better treatment of systemic disease, and improvements in imaging, such as magnetic resonance imaging (MRI), to detect smaller metastases in asymptomatic patients $[1,5,6]$.

The risk of developing BrM varies according to primary tumor type, with lung cancer accounting for approximately one-half of all brain metastases and is likely rising [7, 8]. Two large series have shown the cumulative incidence of BrM to be between 16 and $20 \%$ for lung cancer, $7-10 \%$ for renal cell carcinoma, $7 \%$ for melanoma, $5 \%$ for breast cancer, and $<2 \%$ for colorectal cancer $[9,10]$. One disease in particular, breast cancer, has seen increases in survival even in the metastatic setting. Patients with HER2 overexpression are particularly sensitive to developing BrM, especially those that also have a history of lung metastasis [11]. Longer survival in colorectal cancer has also contributed to the rise in BrM with this diagnosis [12].

The prognosis of patients with brain metastases is poor with the median survival time of untreated patients being approximately one month [13]. With treatment, the overall median survival time after diagnosis is typically still less than one year [14]. Brain metastases present cancer patients with significant neurologic, cognitive, and emotional difficulties and, once diagnosed, generally convey a poor outcome. In this paper, we will review standard treatment approaches with whole brain radiotherapy (WBRT) and discuss new directions in brain metastasis therapy, specifically focusing on the role of radiosurgery and chemical modifiers of the radiation effect. Since neurocognitive dysfunction following brain metastasis is a major concern, we will focus a significant portion of our discussion on this issue.

\section{Diagnosis}

The differential diagnosis for brain metastasis includes infection, paraneoplastic diseases, bleeding, and radiation necrosis (often from previous radiosurgery). As a result biopsies are considered when the diagnosis is uncertain. In fact, in the landmark study from Patchell in colleagues randomizing patients to surgery and whole brain versus biopsy and whole brain, approximately $10 \%$ of patients on the biopsy arm had tumors other than metastasis [15].

On MRI, brain metastases are typically found in the watershed areas of the brain (areas where blood vessels narrow and act as a trap for clumps of tumor cells). Approximately $80 \%$ of the lesions are found in the cerebral hemispheres, $15 \%$ in the cerebellum and $5 \%$ in the brain stem. This is largely 


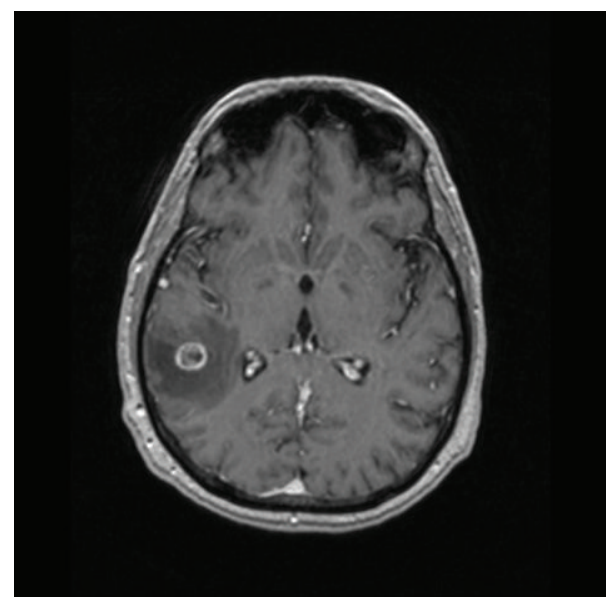

FIGURE 1: T1-weighted brain magnetic resonance imaging scans of a patient with NSCLC: contrast scan showing a $2 \mathrm{~cm}$ right sided parenchymal lesion (courtesy of Sam Chao, MD, Cleveland Clinic Foundation).

based on the general volume of brain in these regions. Areas that are often strategically avoided by therapies, such as the hippocampus, have low propensity for the development of brain metastasis and this has special significance, as will be discussed later [16]. Most of the lesions are intensely enhanced by gadolinium contrast as a result of disruption of the blood brain barrier (see Figure 1). Leptomeningeal metastases, also known as carcinomatous meningitis, are another less common presentation of advanced cancer that occurs when tumor breaks through the arachnoid and pia mater and spreads throughout the subarachnoid space. This is a very aggressive form of metastasis. Symptoms can be quite varied from headaches, to mass effect, to cranial nerve root dysfunction. The most common primary for this to occur is breast cancer but it can occur with other cancers such as lung, melanoma, gastrointestinal tumors, and gynecologic malignancies. MRI is the diagnosis tool of choice and typically one will see enhancement along the walls of the gyri and sulci or multiple nodular deposits throughout the subarachnoid space (see Figure 2). Due to the wide spread dissemination of tumor, WBRT is often employed as part of the treatment strategy for leptomeningeal disease.

\section{Treatment Options with Radiation}

Treatment has evolved over the last several decades. Dexamethasone, for example, has been used to improve symptoms by decreasing capillary permeability and subsequently reducing intracranial edema. A typical dose is $16 \mathrm{mg} /$ day in $2-4$ divided doses. Gastrointestinal prophylaxis with ranitidine or other drugs in its class is often recommended to combat the GI side effects of dexamethasone. For patients on the drug for extended periods of time, thrush prophylaxis is also considered. Steroids are not without side effects as patients can experience weight gain, Cushingoid appearance, proximal muscle weakness, hyperglycemia, fluid retention, and, if tapered to quickly, even adrenal insufficiency. In

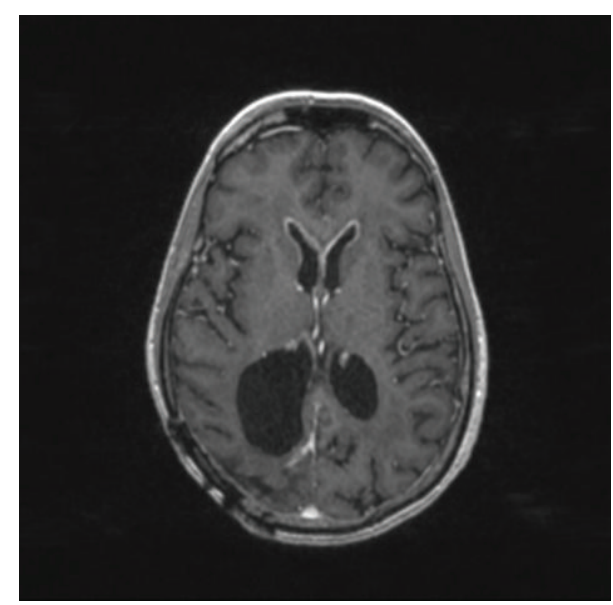

FIGURE 2: A patient with leptomeningeal enhancement (courtesy of Sam Chao, MD, Cleveland Clinic Foundation).

addition to helping alleviate symptoms, steroids were felt to prolong survival from about 1 to 2 months [17].

Traditionally, the most widely used treatment for patients with multiple brain metastases is WBRT. The 1954 article, "Roentgen-ray therapy of cerebral metastases," was one of the earliest reviews of the use of WBRT for the treatment of BrM [32]. The appropriate use of WBRT can provide rapid improvements of many neurologic symptoms, improve quality of life, and may be especially beneficial in patients whose brain metastases are surgically inaccessible or when other medical considerations remove surgery from the list of appropriate options [32-34]. The use of adjuvant WBRT, following resection or radiosurgery has been proven to be effective in terms of improving local control of brain metastases, and, furthermore, the likelihood of neurologic death is decreased based on a randomized trial [35]. The majority of patients who achieve local tumor control die from progression of disease outside of the brain, whereas the cause of death is most often due to central nervous system (CNS) disease in patients with recurrent brain metastases [36]. However, the use of whole brain has come under criticism recently due to the possibility of neurocognitive decline related to brain radiation [37].

Three areas of investigation are attempting to improve the survival of patients with unresectable brain metastases: (1) WBRT in conjunction with radiosurgery (RS), an area that is further explored in other manuscripts in this issue, (2) radiosensitizers, and (3) new chemotherapeutic agents that synergize (or are additive) with radiation. Older studies of radiosensitizers have not resulted in clinical benefit $[38,39]$. Other promising developments in the treatment of brain metastases include new chemotherapy agents such as temozolomide (Temodar), which crosses the blood-brain barrier. Other tumor specific drugs, such as lapatinib (Tykerb), are also being considered for certain primaries, such as HER2 positive breast cancer [40].

3.1. Whole Brain Radiation Therapy. In patients who have brain metastases that impinge upon eloquent areas or are 
TABLE 1: Dose fractionation schemes of WBRT in various tumor types*.

\begin{tabular}{lccc}
\hline Study & Number of patients & Randomization Gy/\# fractions & Median survival (months) \\
\hline Harwood and Simpson [18] & 101 & $30 / 10$ versus $10 / 1$ & $4.0-4.3$ \\
Kurtz et al. [19] & 255 & $30 / 10$ versus 50/20 & $3.9-4.2$ \\
Borgelt et al. [17, 20] & 138 & $10 / 1$ versus 30/10 versus $40 / 20$ & $4.2-4.8$ \\
Borgelt et al. [17, 20] & 64 & $12 / 2$ versus 20/5 & $2.8-3.0$ \\
Chatani et al. [21] & 70 & $30 / 10$ versus 50/20 & $3.0-4.0$ \\
Haie-Meder et al. [22] & 216 & $18 / 3$ versus 36/6 or $43 / 13$ & $4.2-5.3$ \\
Chatani et al. [23] & 72 & $30 / 10$ versus 50/20 or $20 / 5$ & $2.4-4.3$ \\
Murray et al. [24] & 445 & $54.4 / 34$ versus 30/10 & 4.5 \\
\hline
\end{tabular}

* Adapted from Khuntia et al. [25].

TABLE 2: (a) Median survival according to RPA class following WBRT [26]. (b) RTOG Graded Prognostic Assessment (GPA) is felt to be a more sensitive tool for predicting outcomes in BrM patients. They are assigned a score of $0,0.5$, or 1 for each of 4 characteristics. The sum of the score predicts median survival both as a whole (RTOG MS) and with therapy such as WBRT \pm SRS [27]. (c) Disease Specific GPA [28].

(a)

\begin{tabular}{lcccc}
\hline & & \multicolumn{3}{c}{ Median survival (months) by RPA class } \\
Treatment & Patients $(n)$ & $\begin{array}{c}\text { Class 2 } \\
\text { (Primary controlled, age }<65 \text {, no } \\
\text { extracranial metastases, KPS } \geq 70)\end{array}$ & $\begin{array}{c}\text { (primary uncontrolled OR age } \\
\geq 65 \text {, OR extracranial metastases) }\end{array} \quad \begin{array}{c}\text { Class 3 } \\
\text { KPS }<70)\end{array}$ \\
\hline WBRT (RTOG phase III trials) & 1176 & 7.1 & 4.2 & 2.3 \\
\hline
\end{tabular}

(b)

\begin{tabular}{lcccccc}
\hline Score & 0 & 0.5 & 1 & Sum GPA score & RTOG MS & WBRT and/or SRS MS \\
\hline Age & $>60$ & $50-59$ & $<50$ & $3.5-4$ & 11.0 & 21.7 \\
KPS & $<70$ & $70-80$ & $90-100$ & 3 & 6.9 & 17.5 \\
Number brain mets & $>3$ & $2-3$ & 1 & $1.5-2.5$ & 3.8 & 5.9 \\
ExtraCNS mets & Present & - & None & $0-1$ & 2.6 & 3.0 \\
\hline
\end{tabular}

MS = median survival (months).

(c)

\begin{tabular}{lcc}
\hline Primary & $\begin{array}{c}\text { Median survival } \\
\text { (Months) }\end{array}$ & $95 \%$ CI \\
\hline Non-small-cell lung cancer & 7.00 & $6.53-7.50$ \\
Small cell lung cancer & 4.90 & $4.30-6.20$ \\
Melanoma & 6.74 & $5.90-7.57$ \\
Renal cell carcinoma & 9.63 & $7.66-10.91$ \\
Breast cancer & 11.93 & $9.69-12.85$ \\
Gastrointestinal cancer & 5.36 & $4.30-6.30$ \\
Unknown & 6.37 & $5.22-7.49$ \\
\hline
\end{tabular}

too large, numerous, or disseminated for surgery or RS or in patients that performance status does not allow for more aggressive therapy, WBRT alone remains the treatment of choice and provides effective symptom relief in the majority [34]. Response rates following WBRT vary, with complete or partial responses being documented in more than $60 \%$ of patients in randomized controlled studies conducted by the Radiation Therapy Oncology Group (RTOG). Table 1 summarizes results of different dose and fractionation schedules from 8 randomized studies in patients receiving WBRT alone, with median survival ranges from 2.4 to 4.8 months. The consensus from these studies of fractionation schedules is that differences in dose, timing, and fractionation have not significantly altered the median survival time for WBRT treatment of BrM. The landmark RTOG recursive partitioning analysis (RPA) describes three prognostic classes, defined by age, Karnofsky performance status (KPS), and disease status [26]. RPA class 1 patients are younger $(<65)$, have higher KPS scores (70 or greater), no other sites of metastases, and controlled extracranial disease, and have the longest median survival (Table 2(a)).

As treatments for BrM continued to evolve, it became necessary to have additional granularity into the predictive factors associated with BrM to help tailor treatments. The RTOG RPA, for example, was limited in that it did not include the number of lesions. As a result, the RTOG Graded 
Prognostic Assessment (GPA) was developed (Table 2(b)) [27]. In the GPA, data from multiple RTOG studies, including patients from the RTOG 95-08 radiosurgery study, were analyzed and a more sensitive predictive tool was developed that also included number of lesions. With the GPA, primary histology was not included as in the RPA. Therefore, a subsequent disease specific GPA was developed (DS-GPA) (Table 2(c)) [28], but even this system had limitations as we know that the biology is also an important factor in predicting outcomes.

Reports indicate that response to WBRT may be related to the primary histology. Nieder and colleagues [41] studied 108 patients and assessed CT-response based on tumor type following WBRT alone. Complete response was obtained in $24 \%$ of patients and partial response in $35 \%$. Response rates ranged from $81 \%$ for small cell lung carcinoma to $0 \%$ for malignant melanoma (although other series have shown response rates of 45 to $65 \%$ for melanoma brain metastases) [42]. Other retrospective investigations of treatment for brain metastases from various primaries suggest relatively clustered survival statistics: 6 months for female genitourinary cancers [43], 4.2 months for breast cancer [44], 2.3 months [45] for melanoma, and so forth. The RTOG RPA multivariate analysis, however, did not find histology to be an independent predictor of survival following WBRT and this was also factored in the subsequent GPA report.

Another major use of WBRT, which is declining, is as an adjuvant following surgery or radiosurgery. Magnetic resonance imaging reveals that about $80 \%$ of patients have more than 1 metastasis, and $\sim 50 \%$ have 3 or more metastases so treating microscopic disease with WBRT is of value. Furthermore, $70 \%$ of patients with brain metastases experience relapse after resection, if WBRT is omitted [46].

It is important to recognize that there are some arguments against the use of WBRT. Many have questioned its ability to reverse neurologic symptoms [47] and its use has been putatively associated with debilitating complications in long-term survivors $[37,48]$. Furthermore, many patients have short term side effects such as fatigue, alopecia, and erythema. Less common side effects include ear pain, headaches, alterations in smell and taste, and nausea. As a result, the use of WBRT has been declining in the USA.

3.2. Stereotactic Radiosurgery. Traditionally, radiosurgery (SRS) has required the use of Gamma Knife (Elekta, Stockholm, Sweden) radiosurgery system. This is a cobalt based dedicated system used to treat lesions in the brain. As technology has evolved, other manufactures have developed tools such as the Cyberknife (Accuray, Inc., Sunnyvale, CA), Novalis Tx (Varian Medical Systems, Palo Alto, CA and BrainLab, Munich, Germany), TrueBeam (Varian Medical Systems, Palo Alto, CA), and Edge Radiosurgery System (Varian Medical Systems, Palo Alto, CA). Many consider both linac based and cobalt based systems as equivalent and this has allowed for the rapid increase in both intra- and extracranial SRS $[49,50]$.

SRS is now standard practice for patients with 4 or fewer metastases in the brain and this has been validated
TABLE 3: RTOG 9005 [29].

\begin{tabular}{lccccc}
\hline \multicolumn{2}{c}{ Tumor diameter $\leq 20 \mathrm{~mm}$} & \multicolumn{2}{c}{$21-30 \mathrm{~mm}$} & \multicolumn{2}{c}{$31-40 \mathrm{~mm}$} \\
Dose & Toxicity & Dose & Toxicity & Dose & Toxicity \\
\hline $18 \mathrm{~Gy}$ & $8 \%$ & $15 \mathrm{~Gy}$ & $13 \%$ & $12 \mathrm{~Gy}$ & $10 \%$ \\
$21 \mathrm{~Gy}$ & $11 \%$ & $\mathbf{1 8 ~ G y}$ & $20 \%$ & $\mathbf{1 5} \mathrm{Gy}$ & $14 \%$ \\
$\mathbf{2 4 G y}$ & $10 \%$ & $21 \mathrm{~Gy}$ & $38 \%$ & $18 \mathrm{~Gy}$ & $50 \%$ \\
& & $24 \mathrm{~Gy}$ & $58 \%$ & & \\
\hline
\end{tabular}

The MTD for tumors $\leq 20 \mathrm{~mm}$ is $24 \mathrm{~Gy}$, for tumors $21-30 \mathrm{~mm}$ it is $18 \mathrm{~Gy}$, and for tumors $31-40 \mathrm{~mm}$ it is $15 \mathrm{~Gy}$. Doses in yellow represent the recommended MTD.

by multiple prospective trials. The controversy arises when considering its utilization with or without WBRT which is discussed later in detail. The original dose escalation study was reported by Shaw and colleagues in RTOG 9005 where 100 patients with brain metastasis underwent a dose escalation regimen based on the size of the tumor [29]. Smaller tumors were escalated to higher doses (since this could be done safer given the more favorable dosimetry for small lesions) in $3 \mathrm{~Gy}$ increments. The authors concluded that the maximum tolerated dose for tumors $<20 \mathrm{~mm}$ was $24 \mathrm{~Gy}$, between 21 and $30 \mathrm{~mm}$ was $18 \mathrm{~Gy}$, and between 31 and $40 \mathrm{~mm}$ is $15 \mathrm{~Gy}$ (see Table 3).

One of the early efforts reviewing the utility of SRS involved a randomized trial comparing WBRT with or without a SRS boost was done by the University of Pittsburgh. Researchers attempted a phase III study comparing 30 Gy in 12 fractions of WBRT with a 16 Gy boost, but the study was terminated earlier as it was felt unethical to continue given the high control rate in the WBRT + SRS arm (100\%) versus WBRT alone (8\%) [51]. In what is considered one of the defining studies in SRS, the RTOG conducted a 333patient randomized trial comparing WBRT alone (37.5 Gy in 15 fractions) with or without SRS (dosing per RTOG 9005) [30]. The primary endpoint to the study was overall survival with secondary endpoints of tumor response, local control, intracranial recurrence, cause of death, and performance status changes. This study included patients with up to $3 \mathrm{BrM}$. Patients with a single BrM were found to improve in overall survival. The study helped to establish a new standard of care for patients with a single BrM.

The Japanese Radiation Oncology Study Group (JROSG) also conducted a randomized study of SRS versus SRS + WBRT (JROSG 99-1) where 132 patients with up to 4 lesions were randomized [31]. They found that the use of WBRT delayed the occurrence of new brain metastases at 12 months and also reduced the likelihood of the need for salvage brain treatments. The authors concluded that WBRT should not be withheld as intracranial control is paramount (Table 5).

In a provocative study challenging the previous randomized trials mentioned above, Chang and colleagues conducted a single institution randomized trial of SRS with or without WBRT at the MD Anderson Cancer Center [52]. In this study, 58 patients with 1-3 brain metastases (RPA class I or II) were randomized and stratified by RPA class, number of lesions, and radioresistant histologies (melanoma or renal cell versus other). The primary endpoint of the study was 
the Hopkins Verbal Learning Test-Recall (HVLT-R) at 4 months by more than 5 points. Doses used were similar to those in RTOG 95-08 with WBRT given at 30 Gy in 12 fractions. The investigators stopped the study early due to a decreased survival in the WBRT arm (15.2 months versus 5.7 months) which challenged conventional thinking and the data from previous trials (no difference in survival in both RTOG 95-08 and JROSG 99-01). This has come under some criticism in that some argue that the arms were not well balanced as there was more visceral metastatic disease in the WBRT arm and did not take into account subsequent recovery of neurocognitive function as 4 months was the endpoint and that there was a time bias from the fact that WBRT had to be given within 3 weeks after SRS so there could be a 5-week delay in the time points of when the data was analyzed. Given the fact that there were both a reduction of neurocognitive function and also increased death in the WBRT, those that believe in SRS alone have level 1 evidence supporting that decision.

In a European effort, Kocher and colleagues conducted a randomized multi-institutional study comparing adjuvant WBRT versus observation after SRS or surgical resection in patients with 1 to 3 brain metastases [53]. This study randomized a total of 359 patients (199 underwent SRS and 160 underwent resection). After SRS or surgery, adjuvant WBRT was found to reduce intracranial relapse (59\% to $27 \%$ after surgery, $P<0.001$, and $31 \%$ to $19 \%$ after SRS, $P=0.040$ ) and neurologic deaths but did not show improvements in functional independence (10 versus 9.5 months) or overall survival.

Another area of increased interest involves the use of adjuvant SRS after surgical resection. It has been previously noted that the landmark study by Patchell et al. showed the benefits of adjuvant WBRT in improving local control not only in the brain, but also in particular in the site of the resection cavity [35]. However, with the fear of the WBRT toxicities, some have argued that an SRS cavity boost be considered instead of WBRT. In a poll conducted in the 2006 Congress of Neurological Surgeons Meeting, onethird of neurosurgeons resect metastases and follow it by an SRS boost. Soltys and colleagues conducted a retrospective review of 72 patients with 76 cavities from 1998 to 2006 who underwent SRS with a median marginal dose of 18.6 Gy (range of 15-30 Gy) [54]. Actuarial local control was found to be respectable $88 \%$ and $79 \%$ at 6 and 12 months, respectively. This compared favorably to their historical control rate of $54 \%$ for observation alone and $80-90 \%$ with adjuvant WBRT. Furthermore, they recommended using a $2 \mathrm{~mm}$ margin with their technique on the resection cavity. Though this study showed very reasonable results with an SRS cavity boost, other authors have not been able to replicate these results with local control rates as low as 35\% [55]. Part of the poor results may be related to the difficulty in identifying the areas at risk of recurrence due to the difficulty in identifying the postresection cavity. In an effort to clarify this discrepancy, a phase III study is currently under way. In this effort run by the Alliance and endorsed by NRG, NCCTG N107c is currently accruing patients. In this study, patients are randomized to adjuvant whole brain radiation to a dose of 37.5 Gy in 15 fractions versus SRS to the surgical resection bed to a dose between 12 and 20 Gy in a single fraction. Patients will be stratified based on age, duration of controlled extracranial disease, number of preoperative BrM, histology, and size of the resection cavity. The dose in the SRS arm is based on the size of the cavity with the high dose patients exhibiting targets $<4.2 \mathrm{cc}$ receiving $20 \mathrm{~Gy}$ in a single fraction and the low dose region between $\geq 30 \mathrm{cc}$ and $5 \mathrm{~cm}$ max of $12 \mathrm{~Gy}$. The study is expected to accrue a total of 174 eligible patients.

\section{The Controversy Surrounding the Use of Whole Brain Radiotherapy}

With the increased availability of radiosurgery, a new trend has been emerging in the management of patients with brain metastases. In this approach, patients with a limited number of brain metastatic lesions (the exact definition of limited is based on institutional preference and varies from 3 to 10 or more) $[30,31,52,53,56]$ are treated with radiosurgery alone, without WBRT, and are then closely monitored, which involves regular MR imaging. Repeat radiosurgery is performed for new intracranial metastases, with the intent of avoiding or eliminating WBRT in as many patients for as long as possible. The rationale is the avoidance of neurotoxicity from WBRT. However, this approach is still considered controversial by some and definitive controlled clinical trials can support either argument. Delaying WBRT in favor of active surveillance increases the overall cost of managing these patients, with multiple and expensive imaging studies and repeat radiosurgical procedures which also need also needs to be taken into account.

Advocates of withholding WBRT suggest that WBRT may be "ineffective," patients may have "oligometastatic disease," survival is unaltered whether upfront WBRT is used or not, radiosurgery is adequate for local control, and neurologic status and quality of life of patients in whom WBRT is withheld are superior.

Historically, when steroids alone were used for the management of BrM, survival was only about 1 month. The addition of WBRT was found to increase survival by several months. The most common WBRT regimen uses $30 \mathrm{~Gy}$ in 2 weeks; this dose is designed to be palliative and was never intended to cure epithelial tumors but rather strikes a balance of minimizing toxicity but still helping to relieve symptoms. When reviewing data within BrM studies in the RTOG, control of disease is realized in approximately $50 \%$ of patients at 6 months. Therefore, enhancing the effect of WBRT in controlling the macroscopically visible tumor with the addition of radiosurgery makes sense and is now supported by level 1 evidence $[30,31]$. For example, in the phase III RTOG 9508 trial of WBRT with or without radiosurgery boost, the actuarial 1-year local control was 82 versus $71 \%$ $(P=0.01)$, in favor of the radiosurgery boost [30] (Table 4). Furthermore, WBRT can and does add to the local control on top of the SRS treatments as based on both the RTOG and JROSG studies, which does have value.

Some may argue that BrM may be oligometastatic and therefore we only need to worry about the disease that is 
TABLE 4: RTOG 9508 [30]. SRS improved survival in patients with a single BrM. Subset analysis suggested there was a potential benefit for patients with NSCLC, RPA class I, or patients that were $<50$ years old with 2 to 3 metastasis. Patients in the study receiving SRS also had improved KPS with decreased steroid dependence.

\begin{tabular}{lccccc}
\hline Med OS & All pts & Single brain met & $1-3$ mets, age $<50$ & $1-3$ mets, NSCLC & $1-3$ mets, RPA class I \\
\hline WBRT alone & 5.7 mos & 4.9 months & 8.3 months & 3.9 months & 9.6 months \\
WBRT + SRS & 6.5 mos & 6.5 months & 9.9 months & 5.0 months & 11.6 months \\
$P$ value & 0.14 & $\mathbf{0 . 0 4}$ & $\mathbf{0 . 0 4}$ & $\mathbf{0 . 0 5}$ & $\mathbf{0 . 0 5}$ \\
\hline
\end{tabular}

TABLE 5: JROSG 99-1 [31] study comparing SRS with or without WBRT. Omission of WBRT was found to decrease intracranial control, and, as a result, the authors recommended SRS + WBRT for patients with up to 4 brain metastases.

\begin{tabular}{lccccc}
\hline & MS & OS @ 1 yr & Freedom from new brain mets @ 1 mos & Salvage brain tx & Neuro death \\
\hline SRS & 8.0 months & $28.4 \%$ & $46.8 \%$ & $43 \%$ & $19.3 \%$ \\
SRS + WBRT & 7.5 months & $38.5 \%$ & $76.4 \%$ & $<\%$ & $22.8 \%$ \\
$P$ value & 0.42 & 0.42 & $<\mathbf{0 . 0 0 1}$ & $<\mathbf{0 . 0 0 1}$ & 0.64 \\
\hline
\end{tabular}

seen on MR. However, older autopsy and CT-imaging studies suggest that the rate of multiple brain metastases ranges from 58 to $86 \%$, with a mean of $66 \%$, but these have been criticized on several grounds [25]. In one MRI-based study, only $19 \%$ of the 336 patients had a single lesion; the percentage of patients with $2,3,4$, and 5 or more lesions was $16,13,10$, and $40 \%$; in most trials of radiosurgery, $<5$ is considered the upper limit, in terms of the definition of oligometastases, and, in this trial, $50 \%$ of patients had more than 3 lesions on MR imaging [57]. Therefore, most reports suggest that only about $20 \%$ of patients have one brain met, and this is especially important, as evidence-based data suggest no survival benefit from aggressive local treatments such as surgery or radiosurgery in patients with more than 1 metastatic lesion [58].

Others have argued that survival is unaltered whether upfront WBRT is used or not. This is indeed true as there are other competing risks of death, such as systemic disease. However, the value proposition here is that of local control [59]. Many of these studies were not designed to answer an overall survival question as the true value is preventing future complications from recurrent disease and improving or maintaining quality of life [35]. That being said, caution should be used in oversimplifying this statement. For example, Pirzkall et al. reported that, for brain metastases patients without extracranial disease, (i.e., patients with a much lower likelihood of dying from systemic metastases) the median survival following radiosurgery alone with WBRT used for salvage was 8.3 months, compared to 15.4 months for patients treated up-front with radiosurgery plus WBRT [60]. Similar results were seen in a retrospective study from the Mayo Clinic where a survival benefit for adjuvant WBRT limited to patients without systemic disease was realized; 5year survival rates of $21 \%$ for those who received adjuvant WBRT compared to $4 \%$ for those patients who did not [61]. These observations are crucial, implying that, for those patients where prolonged survival is likely, failure to control the intracranial disease by omitting or delaying WBRT may negatively impact survival.

Some have argued that SRS is good enough for local control. In the phase III study, JROSG 99-1, patients were randomized to radiosurgery alone, versus whole brain radiotherapy and radiosurgery [31]. The actuarial 6-month freedom from new brain metastases was $48 \%$ in the SRS alone arm and $82 \%$ in the SRS plus WBRT (log rank, $P=0.003$ ). Actuarial 1-year brain tumor control rate for the lesions treated with radiosurgery was $70 \%$ in the radiosurgery alone arm and $86 \%$ in the radiosurgery and whole brain radiation arm (log rank, $P=0.019$ ) [31]. In another randomized trial [62] comparing SRS alone versus WBRT and SRS versus WBRT, the local brain control rate was highest in the radiosurgery plus WBRT arm. A prospective single arm, multi-institutional ECOG phase II study of radiosurgery alone for "radioresistant" histologies [63] (such as melanoma, sarcoma, and renal cell carcinoma) in patients with 1-3 brain metastases reported at 6 months, 39.2\% failed within the radiosurgery volume, and $39.4 \%$ failed outside the radiosurgery volume, thereby supporting the case of limited benefit from radiosurgery alone. Clinical trial-based assessments therefore suggest high rates of intracranial failures and reduced local control rates when WBRT is omitted or delayed.

Finally, an argument has been made that neurologic status and quality of life of patients in whom WBRT is withheld is superior. In the JRSG 99-01 study, patients were randomized to radiosurgery alone or with WBRT but detailed neurocognitive assessments were not performed, and the primary assessment was by an evaluation of performance status and neurologic functional status, using RTOG criteria [31]. There were no differences in these endpoints between the two study arms, belying the claims of worse neurologic outcomes in the WBRT arm [31]. In fact, some have argued that withholding WBRT increases intracranial failure and neurologic deterioration is more directly related to disease progression in the brain [64]. In a phase III trial of WBRT with or without the radiosensitizer, motexafin gadolinium, the most significant predictor for neurologic and neurocognitive decline, as well as deterioration in quality of life was disease progression in the brain [65].

Therefore, the switch to omitting WBRT is still considered controversial and data supports both withholding and administering WBRT. In large measure, it is fair to say that this switch has been made because physicians have observed some patients experiencing neurocognitive 
and neurologic decline (the causes for which could in fact be multifactorial). This is supported by the Chang study showing a doubling of the rate of neurocognitive decline as a result of WBRT [52]. As a result, efforts, as described below, are underway to help mitigate the toxicities associated with WBRT. Also, multiple studies are underway in single institution phase III studies evaluating the possibility of eliminating WBRT in patients with 5 or more metastases (including UCSF [ClinicalTrials.gov identifier NCT01731704] and MDACC [ClinicalTrials.gov identifier NCT01644591 and NCT01592968]).

4.1. Mechanism of Neurocognitive Dysfunction. Toxicity from radiation can be stratified based on the timing of onset of symptoms: acute, subacute, and late [66]. Acute effects typically occur during the first few weeks of treatment and are often characterized by drowsiness, headache, nausea, vomiting, and worsening focal deficits (typically in the first 30 days). Often, cerebral edema is the cause of these symptoms and corticosteroids such as dexamethasone may improve these symptoms. Subacute symptoms related to encephalopathy (early delayed reaction) occurring at 1 to 6 months after completion of radiation may be secondary to diffuse demyelination $[67,68]$. Symptoms include headache, extreme somnolence, fatigability, and deterioration of preexisting deficits that resolve within several months. Late delayed effects appear more than 6 months after radiation and can be irreversible and progressive [69]. This may be a result of white matter damage due to vascular injury, demyelination, or even necrosis. Symptoms can vary from mild lassitude to significant memory loss and even severe dementia [70]. The pathophysiology of radiation induced neurocognitive damage is complex and multifactorial. Oligodendrocytes, which are important for myelination, are likely at least partially to blame. Oligodendrocyte death can occur either as a result of direct p53 dependent radiation apoptosis or due to exposure to radiation induced tumor necrosis factor $\alpha(\mathrm{TNF} \alpha)[71,72]$. Postradiation injury to the vasculature damages the endothelium leading to platelet aggregation and thrombus formation, followed by abnormal endothelial proliferation and intraluminal collagen deposition [73, 74]. Another area receiving increased attention is hippocampaldependent functions of learning, memory, and spatial information processing that seem to be preferentially affected by radiation [75]. Animal studies reveal that doses as low as $2 \mathrm{~Gy}$ can induce apoptosis in the proliferating cells in the hippocampus leading to decreased repopulative capacity [76].

\subsection{Management and Prevention of Neurocognitive Deficits} from WBRT. Treatment (or prophylaxis) of cognitive sequelae of cranial radiation has been limited until recently. In the past, methylphenidate has been used in a few small series of patients exhibiting neurobehavioral slowing with limited response [77-79]. Patients who develop psychomotor slowing, decline in executive functioning, or general apathy may benefit [78]. Though these studies suggest beneficial effects with methylphenidate, they have significant limitations including small sample size, lack of a blinded control, high toxicity of methylphenidate (including addiction potential), and therefore the widespread use of methylphenidate should not be considered standard of care.

Erythropoietin has been used as a central nervous system protectant in a number of studies and this has generated some interest in the utilization of this agent $[80,81]$. A blinded, randomized trial of erythropoietin (compared to saline) found less motor impairment in erythropoietin treated rats 2 days after $100 \mathrm{~Gy}$ was delivered to the right striatum; by day 10 the erythropoietin treated rats had returned to near control levels while the deficits persisted in the saline treated rats [82]. A similar study found erythropoietin delivered one hour after whole brain radiotherapy (17 Gy in one fraction) was neuroprotective in mice [83]. That being said, currently erythropoietin has not been validated in large human studies and it is not considered a routine part of the management of BrM.

There has been significant interest in using Alzheimer's therapeutic agents to treat radiation induced injury, since many aspects radiation induced injury are clinically and radiographically similar to Alzheimer's dementia. In a trial from Wake Forest University, 24 previously irradiated brain tumor patients were treated with 24 weeks of donepezil [84]. Neurocognitive tests were performed at multiple time points which included tests of verbal fluency, verbal memory, attention, and figural memory scores. Patients were significantly improved between baseline and week 24, but there was no change on global cognitive function or executive function. There was no significant worsening of performance noted on any measures. The limitations of this study include the small sample size and the potential (as well as uncontrolled) impact of practice (i.e., neurocognitive measures repeated over multiple evaluations) and the placebo effect.

Prior studies have suggested that vitamin E may benefit patients with Alzheimer's disease and potentially radiation toxicity [85]. Researchers at the Queen Elizabeth Hospital in Hong Kong treated 19 patients with temporal lobe radionecrosis with a daily megadose of vitamin $\mathrm{E}$ for 1 year, whereas 10 other patients with temporal lobe radionecrosis served as controls (treatment assignment was decided on a voluntary basis) [86]. Significant improvement in global cognitive ability, memory, and executive function occurred among patients in the treatment group after 1-year of vitamin $\mathrm{E}$ treatment. However, there are significant limitations of this study in that the patients were not randomized or blinded to treatment, and therefore the results should be considered hypothesis generating.

Although a neurocognitive conceptual framework for understanding the effects of radiotherapy on the brain is limited [87], it seems that the pathophysiology of late RT injury is dynamic and complex, and a result of interand intracellular interactions between the vasculature and parenchymal compartments and injury is most likely multifactorial (i.e., demyelination, proliferative and degenerative glial reactions, endothelial cell loss, and capillary occlusion) [75]. The vascular hypothesis is the most recognized and longest standing premise as the primary cause of radiation 
induced damage [88]. The vascular hypothesis of radiationinduced injury attributes accelerated atherosclerosis and mineralizing microangiopathy resulting in vascular insufficiency and infarction to radiation injury and inflammation. These mechanisms result in a picture similar to the small vessel disease, as is often seen with vascular dementia and Alzheimer's dementia [89]. For this reason there is interest in using pharmaceutical agents that are effective in the treatment of vascular dementia for irradiated brain tumor patients to serve as a chemoprotectant. One of these agents is memantine, a NMDA receptor antagonist induced by ischemia, which blocks excessive NMDA stimulation leading to excitotoxicity. Agents that block pathologic stimulation of NMDA receptors may protect against further damage in patients with vascular dementia [90]. Thus, NMDA receptor antagonists such as memantine are thought to be a potential neuroprotector when patients are exposed to brain radiation and these drugs will help to prevent neuronal injury associated with radiation-induced ischemia. Furthermore, the physiologic function of the remaining neurons could be restored, resulting in symptomatic improvement [91] and both preclinical in vitro and in vivo data support this hypothesis [92-95]. Phase III clinical trials of memantine in patients with vascular dementia demonstrated clinical benefit, with the subgroup of patients with small-vessel disease responding better to memantine than other types of dementia again, supporting the hypothesis that memantine may function as a neuroprotector $[96,97]$. In addition, there is anecdotal experience using memantine in primary CNS lymphoma patients with cognitive dysfunction after radiation has shown dramatic clinical improvement [98]. With the beneficial findings of these studies and the limitations of treatment of cognitive decline after radiation, the Radiation Therapy Oncology Group (RTOG) has launched and completed a large phase III study comparing patients receiving WBRT to either memantine (Namenda) or placebo (RTOG 0614) [99]. In this landmark study, 508 eligible patients were randomized to the two arms. Patients receiving memantine had less decline in delayed recall at 24 weeks (primary endpoint of the study) though this was not significantly significant. However, this was thought to be partially due to significant patient loss from competing factors such as death from extracranial disease progression. There was better cognitive function over time in many domains of neurocognition, such as memory, executive function, and processing speed. Furthermore, the improved cognition was maintained over time, even when the drug was discontinued, suggesting a protective effect of memantine. Since the drug had relatively few side effects associated with it, the authors concluded that the memantine can be considered along with WBRT when used in the management of BrM. Also of interest in this study is that there are significant biospecimens available for translational research. The investigators are currently analyzing both inflammatory cytokines and biomarkers such as apolipoprotein $\mathrm{E}$ (apoE) as a predictor of patients that are likely to develop neurocognitive decline. Early single institution reports show that those carrying the ApoE allele are more likely to have adverse cognition from radiation [100].
Furthermore, biomarkers can be modulated by the presence of brain radiation [101].

Besides pharmaceutical interventions, others are considering modifying how WBRT is delivered to decrease the risk of neurotoxicity. As mentioned earlier, doses of $2 \mathrm{~Gy}$ or less can damage the hippocampus in the mouse model [76]. However, more recent data has shown that hippocampal dosimetry does indeed predict neurocognitive function impairment. Gondi and colleagues recently reported a prospective study in which 29 patients with benign or low grade brain tumors were treated with fractionated stereotactic radiotherapy. The authors measured the dose to the hippocampi and correlated the outcome to the 18 patients that completed both baseline and 18-month neurocognitive function testing. The study revealed that if that biologically equivalent dose in $2 \mathrm{~Gy}$ fractions exceeded $7.3 \mathrm{~Gy}$, there was a higher likelihood of neurocognitive decline [102]. As a result, current investigations are underway using new technology to conformally avoid the hippocampus. With the use of intensity modulated radiotherapy, it is possible to create isodose distributions that treat the majority of the brain to full dose, while keeping the radiation dose to the hippocampus relatively low. Furthermore, it may be possible to even incorporate a simultaneous boost to the gross disease at the same time (Figure 3 ).

Prior to investigating the possibilities of hippocampal avoidance WBRT (HA-WBRT), it is necessary to identify the likelihood of developing BrM within the hippocampi to ensure that sparing the area would not result in an increase in recurrences. Ghia and colleagues reviewed 100 patients treated with radiation for brain metastasis and found that, of the 100 patients, only 8 had metastases within $5 \mathrm{~mm}$ of the hippocampus [103]. These results were further validated in a larger multi-institutional effort including over 1100 metastases in 371 patients [104]. Less than $9 \%$ of the patients had lesions within $5 \mathrm{~mm}$ of the hippocampus and there were no tumors seen in the hippocampus. As a result, $5 \mathrm{~mm}$ was selected as a safe avoidance region of the hippocampus for a prospective evaluation of HA-WBRT. Furthermore, efforts were made to standardize the technique of how to plan such cases [105].

The major study to date evaluating the effectiveness of HA-WBRT was RTOG 0933 [106]. This study was a single-arm, multi-institutional phase II study evaluating 113 brain metastases patients undergoing HA-WBRT. The study involved a battery of neurocognitive tests (similar to what was seen in RTOG 0614-WBRT with or without memantine) as well as significant credentialing before an institution was able to accrue a patient. Each physician enrolling a patient on the study was required to pass both contouring and planning of a HA-WBRT case. Furthermore, each plan for an enrolled patient underwent central review by RTOG prior to delivery of therapy. Of the 100 analyzable patients, $76 \%$ were categorized as RPA class II. All patients underwent $30 \mathrm{~Gy}$ in 10 fractions of HA-WBRT. The mean dose to the hippocampus was below $10 \mathrm{~Gy}$ in all patients with the maximum dose not exceeding $17 \mathrm{~Gy}$. Patients were assessed using the HVLT-Delayed Recall, (DR) the HVLT-Recall, and the HVLT-Immediate Recognition tests. The primary endpoint of the study was HVLT-DR at 4 months. There were 

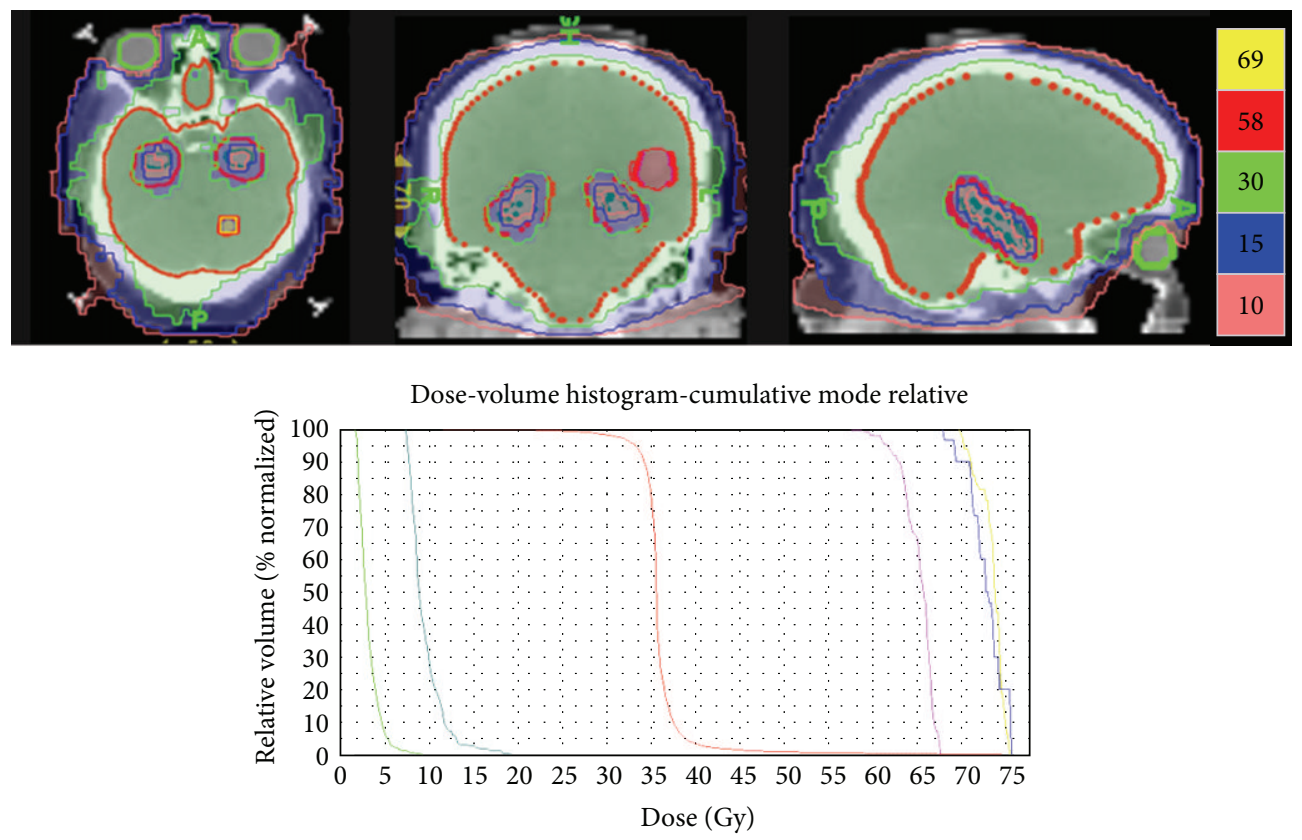

FIGURE 3: Hippocampal sparing whole brain radiotherapy plan showing treatment of the whole brain to 30 Gy while sparing the hippocampi from high doses of radiation which is receiving less than $10 \mathrm{~Gy}$ mean dose. Furthermore, it is also possible to dose escalate the gross disease simultaneously (courtesy of Wolfgang Tomé, Ph.D.).

42 analyzable patients that had four month post-RT data. These patients experienced only a 7\% decline in HVLT-DR which was significantly lower (95\% CI, $-4.7 \%$ to $18.7 \%$ ) of the historical control group of $30 \%$ decline at 4 months. At 6-month follow-up, the decline was even less at $2 \%$, though only 29 patients were analyzable at this point. There were two grade 3 toxicities and no grade 4 or 5 toxicities. The median survival on the study was 6.8 months. As a result of these promising findings, a new phase III study, CC001 has been developed randomizing patients to HA-WBRT versus WBRT. Both study arms will include memantine based on the results seen in RTOG 0614. The study is expected to accrue patients in mid 2015.

\section{Conclusion}

In summary, WBRT continues to be a standard and efficacious treatment in the management of brain metastasis. Despite the use of whole brain radiation and radiosurgery, outcomes are poor and efforts are being made to incorporate multimodality approaches including surgery, radiosurgery, and chemotherapy to improve outcomes. Patients with brain metastasis are susceptible to deficits in neurocognition because of their disease and potentially from the treatment for their brain metastasis. As a result, the use of WBRT is declining despite ample evidence supporting the efficacy. For patients with $>4$ lesions, WBRT still remains the standard of care. For 4 or fewer lesions, SRS alone can be considered but active surveillance for recurrence is needed. Adjuvant SRS to the resection cavity is currently being evaluated in a randomized trial and remains a study question.
Innovative strategies for preventing and treating neurocognitive deficits are actively under investigation and include chemoprevention with drugs such as memantine, but also the use of advanced radiation delivery techniques to spare regions of the brain susceptible to radiation injury to reduce the likelihood of neurocognitive deficits. Phase II studies suggest a strong benefit for HA-WBRT. Many of the recently closed and currently open cooperative group trials have extensive translational components as part of the studies. It is the hope that biomarkers may be able to predict which patients are likely to develop neurocognitive decline and which ones will not, even in the presence of WBRT. These types of study will allow clinicians to personalize the WBRT and SRS treatment strategies for the patients they see.

\section{Conflict of Interests}

In addition to being a practicing Radiation Oncologist, Dr. Khuntia serves as the Vice-President of Medical Affairs at Varian Medical Systems, a leading manufacturer of radiation oncology hardware and software.

\section{Acknowledgments}

The author would like to offer special thanks to Minesh Mehta, MD, at the University of Maryland, Wolfgang Tomé, Ph.D., from Albert Einstein University, Vinai Gondi, MD, from Cadence Health, and Paul Brown, MD, from MD Anderson Cancer center for their years of support in the advancement of technology and delivery of radiation for the care of patients with brain metastases. 


\section{References}

[1] P. Y. Wen, P. M. Black, and J. S. Loeffler, "Metastatic brain cancer," in Cancer: Principles and Practice of Oncology, V. DeVita, S. Hellman, and S. A. Rosenberg, Eds., pp. 2655-2670, Lippincott, WIlliams, \& Wilkins, Philadelphia, Pa, USA, 6th edition, 2001.

[2] A. D. Norden, P. Y. Wen, and S. Kesari, "Brain metastases," Current Opinion in Neurology, vol. 18, no. 6, pp. 654-661, 2005.

[3] M. P. Mehta and I. Tremont-Lukats, "Radiosurgery for single and mutliple brain metastasis," in Intracranial Metastases: Current Management Strategies, R. Sawaya, Ed., pp. 139-164, Futura Division, Blackwell Publishing, Malden, Mass, USA, 2004.

[4] H. Greenberg, W. F. Chandler, and H. M. Sandler, "Brain metastases," in Brain Tumors, H. Greenberg, W. F. Chandler, and H. M. Sandler, Eds., pp. 299-317, Oxford University Press, New York, NY, USA, 1999.

[5] M. L. Sundermeyer, N. J. Meropol, A. Rogatko, H. Wang, and S. J. Cohen, "Changing patterns of bone and brain metastases in patients with colorectal cancer," Clinical Colorectal Cancer, vol. 5, no. 2, pp. 108-113, 2005.

[6] J. D. Johnson and B. Young, "Demographics of brain metastasis," Neurosurgery Clinics of North America, vol. 7, no. 3, pp. 337-344, 1996.

[7] B. P. Yawn, P. C. Wollan, C. Schroeder, L. Gazzuola, and M. Mehta, "Temporal and gender-related trends in brain metastases from lung and breast cancer," Minnesota Medicine, vol. 86, no. 12, pp. 32-37, 2003.

[8] H. J. Mamon, B. Y. Yeap, P. A. Jänne et al., "High risk of brain metastases in surgically staged IIIA non-small-cell lung cancer patients treated with surgery, chemotherapy, and radiation," Journal of Clinical Oncology, vol. 23, no. 7, pp. 1530-1537, 2005.

[9] J. S. Barnholtz-Sloan, A. E. Sloan, F. G. Davis, F. D. Vigneau, P. Lai, and R. E. Sawaya, "Incidence proportions of brain metastases in patients diagnosed (1973 to 2001) in the Metropolitan Detroit Cancer Surveillance System," Journal of Clinical Oncology, vol. 22, no. 14, pp. 2865-2872, 2004.

[10] L. J. Schouten, J. Rutten, H. A. M. Huveneers, and A. Twijnstra, "Incidence of brain metastases in a cohort of patients with carcinoma of the breast, colon, kidney, and lung and melanoma," Cancer, vol. 94, no. 10, pp. 2698-2705, 2002.

[11] K. Slimane, F. Andre, S. Delaloge et al., "Risk factors for brain relapse in patients with metastatic breast cancer," Annals of Oncology, vol. 15, no. 11, pp. 1640-1644, 2004.

[12] T. J. Kruser, S. T. Chao, P. Elson et al., "Multidisciplinary management of colorectal brain metastases: a retrospective study," Cancer, vol. 113, no. 1, pp. 158-165, 2008.

[13] S. Zimm, G. L. Wampler, D. Stablein, T. Hazra, and H. F. Young, "Intracerebral metastases in solid-tumor patients: natural history and results of treatment," Cancer, vol. 48, no. 2, pp. 384394, 1981.

[14] J. T. Sundström, H. Minn, K. K. Lertola, and E. Nordman, "Prognosis of patients treated for intracranial metastases with whole-brain irradiation," Annals of Medicine, vol. 30, no. 3, pp. 296-299, 1998.

[15] R. A. Patchell, P. A. Tibbs, J. W. Walsh et al., "A randomized trial of surgery in the treatment of single metastases to the brain," The New England Journal of Medicine, vol. 322, no. 8, pp. 494-500, 1990.

[16] V. Gondi, W. A. Tomé, and M. P. Mehta, "Why avoid the hippocampus? A comprehensive review," Radiotherapy and Oncology, vol. 97, no. 3, pp. 370-376, 2010.
[17] B. Borgelt, R. Gelber, M. Larson, F. Hendrickson, T. Griffin, and R. Roth, "Ultra-rapid high dose irradiation schedules for the palliation of brain metastases: final results of the first two studies by the radiation therapy oncology group," International Journal of Radiation Oncology, Biology, Physics, vol. 7, no. 12, pp. 1633$1638,1981$.

[18] A. R. Harwood and W. J. Simpson, "Radiation therapy of cerebral metastases: a randomized prospective clinical trial," International Journal of Radiation Oncology Biology Physics, vol. 2, no. 11-12, pp. 1091-1094, 1977.

[19] J. M. Kurtz, R. Gelber, L. W. Brady, R. J. Carella, and J. S. Cooper, "The palliation of brain metastases in a favorable patient population: a randomized clinical trial by the radiation therapy oncology group," International Journal of Radiation Oncology, Biology, Physics, vol. 7, no. 7, pp. 891-895, 1981.

[20] B. Borgelt, R. Gelber, S. Kramer et al., "The palliation of brain metastases: final results of the first two studies by the radiation therapy oncology group," International Journal of Radiation Oncology, Biology, Physics, vol. 6, no. 1, pp. 1-9, 1980.

[21] M. Chatani, T. Teshima, K. Hata, and T. Inoue, "Prognostic factors in patients with brain metastases from lung carcinoma," Strahlentherapie und Onkologie, vol. 162, no. 3, pp. 157-161, 1986.

[22] C. Haie-Meder, B. Pellae-Cosset, A. Laplanche et al., "Results of a randomized clinical trial comparing two radiation schedules in the palliative treatment of brain metastases," Radiotherapy and Oncology, vol. 26, no. 2, pp. 111-116, 1993.

[23] M. Chatani, Y. Matayoshi, N. Masaki, and T. Inoue, "Radiation therapy for brain metastases from lung carcinoma. Prospective randomized trial according to the level of lactate dehydrogenase," Strahlentherapie und Onkologie, vol. 170, no. 3, pp. 155$161,1994$.

[24] K. J. Murray, C. Scott, H. M. Greenberg et al., "A randomized phase III study of accelerated hyperfractionation versus standard in patients with unresected brain metastases: A report of the Radiation Therapy Oncology Group (RTOG) 9104," International Journal of Radiation, Oncology, Biology, Physics, vol. 39, no. 3, pp. 571-574, 1997.

[25] D. Khuntia, P. Brown, J. Li, and M. P. Mehta, "Whole-brain radiotherapy in the management of brain metastasis," Journal of Clinical Oncology, vol. 24, no. 8, pp. 1295-1304, 2006.

[26] L. Gaspar, C. Scott, M. Rotman et al., "Recursive Partitioning Analysis (RPA) of prognostic factors in three Radiation Therapy Oncology Group (RTOG) brain metastases trials," International Journal of Radiation Oncology Biology Physics, vol. 37, no. 4, pp. 745-751, 1997.

[27] C. M. Sperduto, Y. Watanabe, J. Mullan et al., "A validation study of a new prognostic index for patients with brain metastases: the Graded Prognostic Assessment," Journal of Neurosurgery, vol. 109, pp. 87-89, 2008.

[28] P. W. Sperduto, S. T. Chao, P. K. Sneed et al., "Diagnosisspecific prognostic factors, indexes, and treatment outcomes for patients with newly diagnosed brain metastases: a multiinstitutional analysis of 4,259 patients," International Journal of Radiation Oncology Biology Physics, vol. 77, no. 3, pp. 655-661, 2010.

[29] E. Shaw, C. Scott, L. Souhami et al., "Single dose radiosurgical treatment of recurrent previously irradiated primary brain tumors and brain metastases: final report of RTOG protocol 9005," International Journal of Radiation Oncology Biology Physics, vol. 47, no. 2, pp. 291-298, 2000.

[30] D. W. Andrews, C. B. Scott, P. W. Sperduto et al., "Whole brain radiation therapy with or without stereotactic radiosurgery 
boost for patients with one to three brain metastases: phase III results of the RTOG 9508 randomised trial," The Lancet, vol. 363, no. 9422, pp. 1665-1672, 2004.

[31] H. Aoyama, H. Shirato, M. Tago et al., "Stereotactic radiosurgery plus whole-brain radiation therapy vs stereotactic radiosurgery alone for treatment of brain metastases: a randomized controlled trial," The Journal of the American Medical Association, vol. 295, no. 21, pp. 2483-2491, 2006.

[32] J. H. Chao, R. Phillips, and J. J. Nickson, "Roentgen-ray therapy of cerebral metastases,” Cancer, vol. 7, no. 4, pp. 682-689, 1954.

[33] S. E. Order, S. Hellman, C. F. von Essen, and M. M. Kligerman, "Improvement in quality of survival following whole-brain irradiation for brain metastasis," Radiology, vol. 91, no. 1, pp. 149-153, 1968.

[34] L. R. Coia, "The role of radiation therapy in the treatment of brain metastases," International Journal of Radiation Oncology Biology Physics, vol. 23, no. 1, pp. 229-238, 1992.

[35] R. A. Patchell and W. F. Regine, "The rationale for adjuvant whole brain radiation therapy with radiosurgery in the treatment of single brain metastases," Technology in Cancer Research and Treatment, vol. 2, no. 2, pp. 111-115, 2003.

[36] E. Arbit, M. Wroński, M. Burt, and J. H. Galicich, "The treatment of patients with recurrent brain metastases. A retrospective analysis of 109 patients with nonsmall cell lung cancer," Cancer, vol. 76, no. 5, pp. 765-773, 1995.

[37] M. Mehta, "The dandelion effect: treat the whole lawn or weed selectively?" Journal of Clinical Oncology, vol. 29, no. 2, pp. 121124, 2011.

[38] M. D. Prados, C. B. Scott, M. Rotman et al., "Influence of bromodeoxyuridine radiosensitization on malignant glioma patient survival: a retrospective comparison of survival data from the Northern California Oncology Group (NCOG) and Radiation Therapy Oncology Group Trials (RTOG) for glioblastoma multiforme and anaplastic astrocytoma," International Journal of Radiation Oncology Biology Physics, vol. 40, no. 3, pp. 653-659, 1998.

[39] M. D. Prados, W. Seiferheld, H. M. Sandler et al., "Phase III randomized study of radiotherapy plus procarbazine, lomustine, and vincristine with or without BUdR for treatment of anaplastic astrocytoma: final report of RTOG 9404," International Journal of Radiation Oncology Biology Physics, vol. 58, no. 4, pp. 1147-1152, 2004.

[40] C. Arslan, O. Dizdar, and K. Altundag, "Chemotherapy and biological treatment options in breast cancer patients with brain metastasis: an update," Expert Opinion on Pharmacotherapy, vol. 15, no. 12, pp. 1643-1658, 2014.

[41] C. Nieder, W. Berberich, and K. Schnabel, “Tumor-related prognostic factors for remission of brain metastases after radiotherapy," International Journal of Radiation Oncology Biology Physics, vol. 39, no. 1, pp. 25-30, 1997.

[42] J. Ellerhorst, E. Strom, E. Nardone, and I. McCutcheon, "Whole brain irradiation for patients with metastatic melanoma: a review of 87 cases," International Journal of Radiation Oncology Biology Physics, vol. 49, no. 1, pp. 93-97, 2001.

[43] A. S. Mahmoud-Ahmed, P. A. Kupelian, C. A. Reddy, and J. H. Suh, "Brain metastases from gynecological cancers: factors that affect overall survival," Technology in Cancer Research and Treatment, vol. 1, no. 4, pp. 305-310, 2002.

[44] A. S. Mahmoud-Ahmed, J. H. Suh, S.-Y. Lee, R. L. Crownover, and G. H. Barnett, "Results of whole brain radiotherapy in patients with brain metastases from breast cancer: a retrospective study," International Journal of Radiation Oncology Biology Physics, vol. 54, no. 3, pp. 810-817, 2002.

[45] J. C. Buchsbaum, J. H. Suh, S.-Y. Lee, M. A. Chidel, J. F. Greskovich, and G. H. Barnett, "Survival by radiation therapy oncology group recursive partitioning analysis class and treatment modality in patients with brain metastases from malignant melanoma: a retrospective study," Cancer, vol. 94, no. 8, pp. 2265-2272, 2002.

[46] R. A. Patchell, P. A. Tibbs, W. F. Regine et al., "Postoperative radiotherapy in the treatment of single metastases to the brain: a randomized trial," Journal of the American Medical Association, vol. 280, no. 17, pp. 1485-1489, 1998.

[47] A. Bezjak, J. Adam, R. Barton et al., "Symptom response after palliative radiotherapy for patients with brain metastases," European Journal of Cancer, vol. 38, no. 4, pp. 487-496, 2002.

[48] L. M. DeAngelis, J.-Y. Delattre, and J. B. Posner, "Radiationinduced dementia in patients cured of brain metastases," Neurology, vol. 39, no. 6, pp. 789-796, 1989.

[49] V. W. Stieber, J. D. Bourland, W. A. Tomé, and M. P. Mehta, "Gentlemen (and ladies), choose your weapons: gamma Knife vs. linear accelerator radiosurgery," Technology in Cancer Research and Treatment, vol. 2, no. 2, pp. 79-85, 2003.

[50] E. M. Thomas, R. A. Popple, X. Wu et al., "Comparison of plan quality and delivery time between volumetric arc therapy (rapidarc) and gamma knife radiosurgery for multiple cranial metastases," Neurosurgery, vol. 75, no. 4, pp. 409-418, 2014.

[51] D. Kondziolka, A. Patel, L. D. Lunsford, A. Kassam, and J. C. Flickinger, "Stereotactic radiosurgery plus whole brain radiotherapy versus radiotherapy alone for patients with multiple brain metastases," International Journal of Radiation Oncology Biology Physics, vol. 45, no. 2, pp. 427-434, 1999.

[52] E. L. Chang, J. S. Wefel, K. R. Hess et al., "Neurocognition in patients with brain metastases treated with radiosurgery or radiosurgery plus whole-brain irradiation: a randomised controlled trial," The Lancet Oncology, vol. 10, no. 11, pp. 10371044, 2009.

[53] M. Kocher, R. Soffietti, U. Abacioglu et al., "Adjuvant wholebrain radiotherapy versus observation after radiosurgery or surgical resection of one to three cerebral metastases: results of the EORTC 22952-26001 study," Journal of Clinical Oncology, vol. 29, no. 2, pp. 134-141, 2011.

[54] S. G. Soltys, J. R. Adler, J. D. Lipani et al., "Stereotactic radiosurgery of the postoperative resection cavity for brain metastases," International Journal of Radiation Oncology Biology Physics, vol. 70, no. 1, pp. 187-193, 2008.

[55] A. Narayana and K. Chan, "A phase II trial of stereotactic radiosurgery boost following surgical resection for solitary brain metastases," Journal of Clinical Oncology, vol. 24, p. 71S, 2006, ASCO Abstract.

[56] W. S. Chang, H. Y. Kim, J. W. Chang, Y. G. Park, and J. H. Chang, "Analysis of radiosurgical results in patients with brain metastases according to the number of brain lesions: is stereotactic radiosurgery effective for multiple brain metastases?" Journal of Neurosurgery, vol. 113, supplement, pp. 73-78, 2010.

[57] G. Sze, M. Mehta, C. J. Schultz et al., Radiologic Response Evaluation of Brain Metastases: Uni-Dimensional (1D) W.H.O. Recist vs. Bi-Dimensional (2D) Or 3-Dimensional (3D) Criteria, American Society of Clinical Oncology, 2001.

[58] M. P. Mehta, M. N. Tsao, T. J. Whelan et al., "The American Society for Therapeutic Radiology and Oncology (ASTRO) 
evidence-based review of the role of radiosurgery for brain metastases," International Journal of Radiation Oncology Biology Physics, vol. 63, no. 1, pp. 37-46, 2005.

[59] C. J. Langer and M. P. Mehta, "Current management of brain metastases, with a focus on systemic options," Journal of Clinical Oncology, vol. 23, no. 25, pp. 6207-6219, 2005.

[60] A. Pirzkall, J. Debus, F. Lohr et al., "Radiosurgery alone or in combination with whole-brain radiotherapy for brain metastases," Journal of Clinical Oncology, vol. 16, no. 11, pp. 3563-3569, 1998.

[61] S. R. Smalley, E. R. Laws Jr., J. R. O’Fallon, E. G. Shaw, and M. F. Schray, "Resection for solitary brain metastasis. Role of adjuvant radiation and prognostic variables in 229 patients," Journal of Neurosurgery, vol. 77, no. 4, pp. 531-540, 1992.

[62] P. B. Chougule, M. Burton-Williams, S. Saris et al., "Randomized treatment of brain metastasis with gamma knife radiosurgery, whole brain radiotherapy or both," International Journal of Radiation Oncology, Biology, Physics, vol. 48, no. 3, supplement 1, p. 114, 2000.

[63] R. Manon, A. O’Neill, J. Knisely et al., "Phase II trial of radiosurgery for one to three newly diagnosed brain metastases from renal cell carcinoma, melanoma, and sarcoma: an eastern cooperative oncology group study (E 6397)," Journal of Clinical Oncology, vol. 23, no. 34, pp. 8870-8876, 2005.

[64] W. F. Regine, J. L. Huhn, R. A. Patchell et al., "Risk of symptomatic brain tumor recurrence and neurologic deficit after radiosurgery alone in patients with newly diagnosed brain metastases: results and implications," International Journal of Radiation Oncology, Biology, Physics, vol. 52, no. 2, pp. 333-338, 2002.

[65] C. A. Meyers, J. A. Smith, A. Bezjak et al., "Neurocognitive function and progression in patients with brain metastases treated with whole-brain radiation and motexafin gadolinium: results of a randomized phase III trial," Journal of Clinical Oncology, vol. 22, no. 1, pp. 157-165, 2004.

[66] G. E. Sheline, W. M. Wara, and V. Smith, "Therapeutic irradiation and brain injury," International Journal of Radiation Oncology Biology Physics, vol. 6, no. 9, pp. 1215-1228, 1980.

[67] A. J. van der Kogel, "Radiation-induced damage in the central nervous system: an interpretation of target cell responses," British Journal of Cancer, vol. 53, no. 7, pp. 207-217, 1986.

[68] E. Boldrey and G. Sheline, "Delayed transitory clinical manifestations after radiation treatment of intracranial tumors," Acta Radiologica: Therapy, Physics, Biology, vol. 5, pp. 5-10, 1966.

[69] S. Kramer, "The hazards of therapeutic iradiation of the central nervous system," Clinical neurosurgery, vol. 15, pp. 301-318, 1968.

[70] T. E. Schultheiss, L. E. Kun, K. K. Ang, and L. C. Stephens, "Radiation response of the central nervous system," International Journal of Radiation Oncology, Biology, Physics, vol. 31, no. 5, pp. 1093-1112, 1995.

[71] B. M. Chow, Y. Q. Li, and C. S. Wong, "Radiation-induced apoptosis in the adult central nervous system is p53-dependent," Cell Death and Differentiation, vol. 7, no. 8, pp. 712-720, 2000.

[72] W. Cammer, "Effects of TNF $\alpha$ on immature and mature oligodendrocytes and their progenitors in vitro," Brain Research, vol. 864, no. 2, pp. 213-219, 2000.

[73] J. R. Crossen, D. Garwood, E. Glatstein, and E. A. Neuwelt, "Neurobehavioral sequelae of cranial irradiation in adults: a review of radiation-induced encephalopathy," Journal of Clinical Oncology, vol. 12, no. 3, pp. 627-642, 1994.
[74] P. C. Burger, M. S. Mahaley Jr., L. Dudka, and F. S. Vogel, “The morphologic effects of radiation administered therapeutically for intracranial gliomas. A postmortem study of 25 cases," Cancer, vol. 44, no. 4, pp. 1256-1272, 1979.

[75] M. L. Monje and T. Palmer, "Radiation injury and neurogenesis," Current Opinion in Neurology, vol. 16, no. 2, pp. 129-134, 2003.

[76] W. Peißner, M. Kocher, H. Treuer, and F. Gillardon, "Ionizing radiation-induced apoptosis of proliferating stem cells in the dentate gyrus of the adult rat hippocampus," Molecular Brain Research, vol. 71, no. 1, pp. 61-68, 1999.

[77] R. DeLong, H. Friedman, N. Friedman, K. Gustafson, and J. Oakes, "Methylphenidate in neuropsychological sequelae of radiotherapy and chemotherapy of childhood brain tumors and leukemia," Journal of Child Neurology, vol. 7, no. 4, pp. 462-463, 1992.

[78] M. A. Weitzner, C. A. Meyers, and A. D. Valentine, "Methylphenidate in the treatment of neurobehavioral slowing associated with cancer and cancer treatment," Journal of Neuropsychiatry and Clinical Neurosciences, vol. 7, no. 3, pp. 347-350, 1995.

[79] C. A. Meyers, M. A. Weitzner, A. D. Valentine, and V. A. Levin, "Methylphenidate therapy improves cognition, mood, and function of brain tumor patients," Journal of Clinical Oncology, vol. 16, no. 7, pp. 2522-2527, 1998.

[80] S. A. Lipton, "Erythropoietin for neurologic protection and diabetic neuropathy," The New England Journal of Medicine, vol. 350, no. 24, pp. 2516-2517, 2004.

[81] H. Ehrenreich, M. Hasselblatt, C. Dembowski et al., "Erythropoietin therapy for acute stroke is both safe and beneficial," Molecular Medicine, vol. 8, no. 8, pp. 495-505, 2002.

[82] J. P. Knisely, A. C. de Lotbiniere, N. C. de Lanerolle, and M. L. Brines, "Randomized trial of erythropoietin as a central nervous system radioprotectant," International Journal of Radiation Oncology, Biology, Physics, vol. 60, no. 1, supplement, pp. S343S344, 2004.

[83] M. Hossain and C. S. Wong, "Abstract 3125: erythropoietin improves learning and memory impairment after whole brain irradiation," Cancer Research, vol. 64, p. 724, 2004.

[84] S. R. Rapp, R. Rosdhal, R. D’Agostino et al., "Phase II study of Ginkgo biloba in irradiated brain tumor patients: effect on cognitive function, quality of life, and mood," Journal of NeuroOncology, vol. 109, no. 2, pp. 357-363, 2012.

[85] M. Sano, C. Ernesto, R. G. Thomas et al., "A controlled trial of selegiline, alpha-tocopherol, or both as treatment for Alzheimer's disease," The New England Journal of Medicine, vol. 336, no. 17, pp. 1216-1222, 1997.

[86] A. S. Chan, M.-C. Cheung, S. C. Law, and J. H. Chan, "Phase II study of alpha-tocopherol in improving the cognitive function of patients with temporal lobe radionecrosis," Cancer, vol. 100, no. 2, pp. 398-404, 2004.

[87] C. L. Armstrong, K. Gyato, A. W. Awadalla, R. Lustig, and Z. A. Tochner, "A critical review of the clinical effects of therapeutic irradiation damage to the brain: the roots of controversy," Neuropsychology Review, vol. 14, no. 1, pp. 65-86, 2004.

[88] S. B. Wolbach, "The pathologic history of chronic X-ray dermatitis \& early X-ray carcinoma," The Journal of Medical Research, vol. 21, pp. 415-449, 1909.

[89] C. Belka, W. Budach, R. D. Kortmann, and M. Bamberg, "Radiation induced CNS toxicity-molecular and cellular mechanisms," British Journal of Cancer, vol. 85, no. 9, pp. 1233-1239, 2001. 
[90] E. Lancelot and M. F. Beal, "Glutamate toxicity in chronic neurodegenerative disease," Progress in Brain Research, vol. 116, pp. 331-347, 1998.

[91] J. Kornhuber, M. Weller, K. Schoppmeyer, and P. Riederer, "Amantadine and memantine are NMDA receptor antagonists with neuroprotective properties," Journal of Neural Transmission Supplementum, no. 43, pp. 91-104, 1994.

[92] J. W. Pellegrini and S. A. Lipton, "Delayed administration of memantine prevents $\mathrm{N}$-methyl-D-aspartate receptor-mediated neurotoxicity," Annals of Neurology, vol. 33, no. 4, pp. 403-407, 1993.

[93] H. S. V. Chen, J. W. Pellegrini, S. K. Aggarwal et al., "Openchannel block of N-methyl-D-aspartate (NMDA) responses by memantine: therapeutic advantage against NMDA receptormediated neurotoxicity," The Journal of Neuroscience, vol. 12, no. 11, pp. 4427-4436, 1992.

[94] H.-S. V. Chen and S. A. Lipton, "Mechanism of memantine block of NMDA-activated channels in rat retinal ganglion cells: uncompetitive antagonism," The Journal of Physiology, vol. 499, no. 1, pp. 27-46, 1997.

[95] H.-S. V. Chen, Y. F. Wang, P. V. Rayudu et al., "Neuroprotective concentrations of the N-methyl-D-aspartate openchannel blocker memantine are effective without cytoplasmic vacuolation following post-ischemic administration and do not block maze learning or long-term potentiation," Neuroscience, vol. 86, no. 4, pp. 1121-1132, 1998.

[96] J.-M. Orgogozo, A.-S. Rigaud, A. Stöffler, H.-J. Möbius, and F. Forette, "Efficacy and safety of memantine in patients with mild to moderate vascular dementia: a randomized, placebocontrolled trial (MMM 300)," Stroke, vol. 33, no. 7, pp. 18341839, 2002.

[97] G. Wilcock, H. J. Möbius, and A. Stöffler, "A double-blind, placebo-controlled multicentre study of memantine in mild to moderate vascular dementia (MMM500)," International Clinical Psychopharmacology, vol. 17, no. 6, pp. 297-305, 2002.

[98] M. P. Mehta and I. Robbins, "Improvement in cognitive function with memantine in patient with PCNSL and cognitive impairment after therapy," Personal Communication. In press.

[99] P. D. Brown, S. Pugh, N. N. Laack et al., "Memantine for the prevention of cognitive dysfunction in patients receiving whole-brain radiotherapy: a randomized, double-blind, placebo-controlled trial," Neuro-Oncology, vol. 15, no. 10, pp. 1429-1437, 2013.

[100] D. D. Correa, J. Satagopan, R. E. Baser et al., "APOE polymorphisms and cognitive functions in patients with brain tumors," Neurology, vol. 83, no. 4, pp. 320-327, 2014.

[101] M. Kalm, E. Abel, P. Wasling et al., "Neurochemical evidence of potential neurotoxicity after prophylactic cranial irradiation," International Journal of Radiation Oncology, Biology, Physics, vol. 89, no. 3, pp. 607-614, 2014.

[102] V. Gondi, B. P. Hermann, M. P. Mehta, and W. A. Tomé, "Hippocampal dosimetry predicts neurocognitive function impairment after fractionated stereotactic radiotherapy for benign or low-grade adult brain tumors," International Journal of Radiation Oncology, Biology, Physics, vol. 85, no. 2, pp. 348354, 2013

[103] A. Ghia, W. A. Tomé, S. Thomas et al., "Distribution of brain metastases in relation to the hippocampus: implications for neurocognitive functional preservation," International Journal of Radiation Oncology Biology Physics, vol. 68, no. 4, pp. 971977, 2007.
[104] V. Gondi, W. A. Tome, J. Marsh et al., "Estimated risk of perihippocampal disease progression after hippocampal avoidance during whole-brain radiotherapy: safety profile for RTOG 0933," Radiotherapy and Oncology, vol. 95, no. 3, pp. 327-331, 2010.

[105] V. Gondi, R. Tolakanahalli, M. P. Mehta et al., "Hippocampalsparing whole-brain radiotherapy: a 'how-to' technique using helical tomotherapy and linear accelerator-based intensitymodulated radiotherapy," International Journal of Radiation Oncology Biology Physics, vol. 78, no. 4, pp. 1244-1252, 2010.

[106] V. Gondi, S. L. Pugh, W. A. Tome et al., "Preservation of memory with conformal avoidance of the hippocampal neural stemcell compartment during whole-brain radiotherapy for brain metastases (RTOG 0933): a phase II multi-institutional trial," Journal of Clinical Oncology, vol. 32, no. 34, pp. 3810-3816, 2014. 

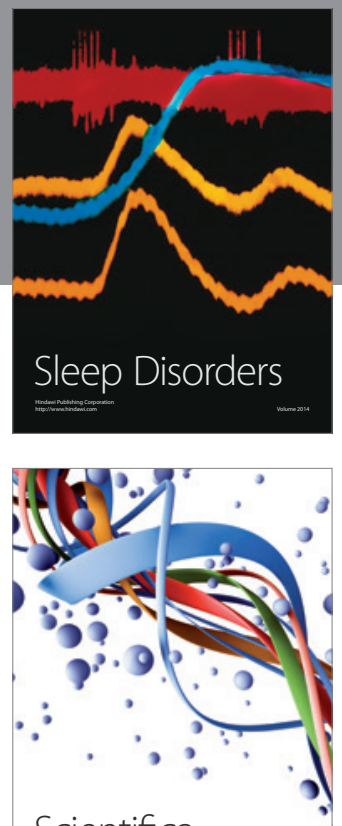

Scientifica
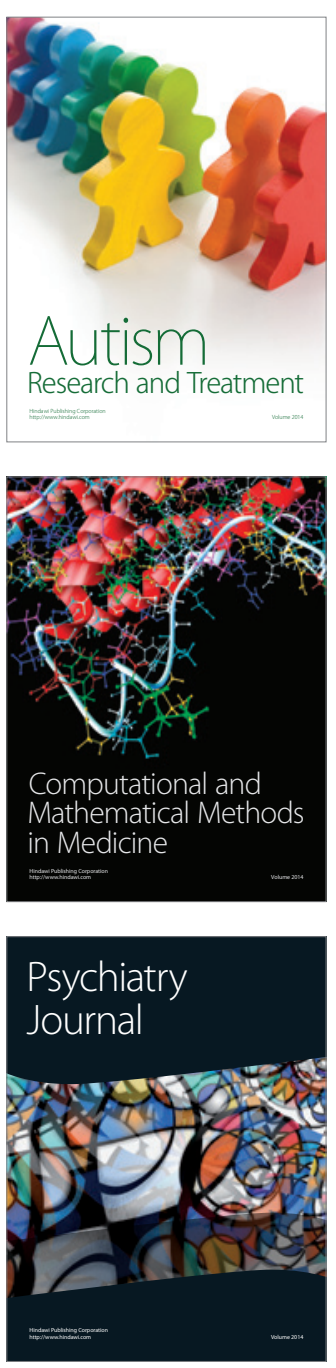
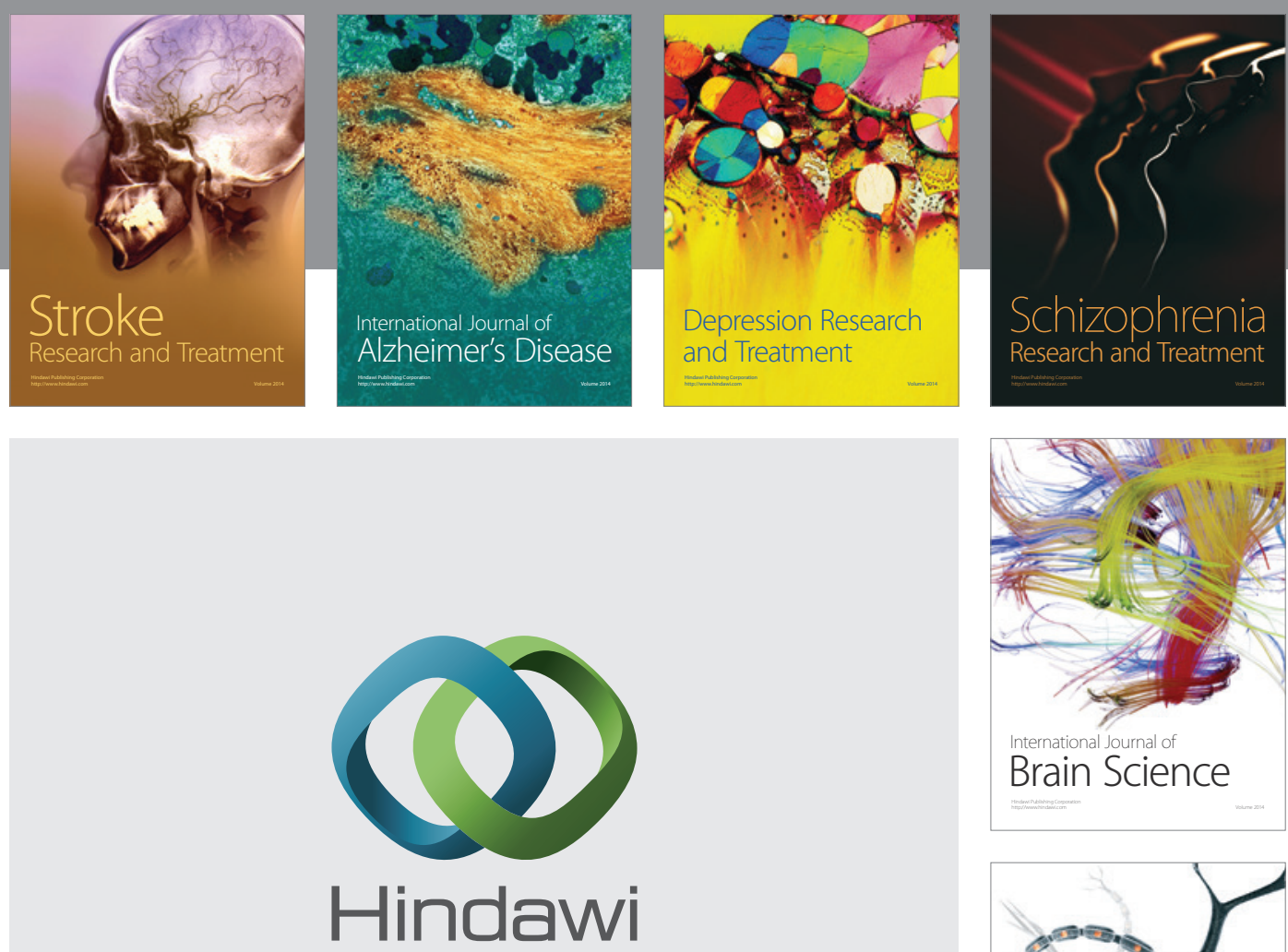

Submit your manuscripts at

http://www.hindawi.com
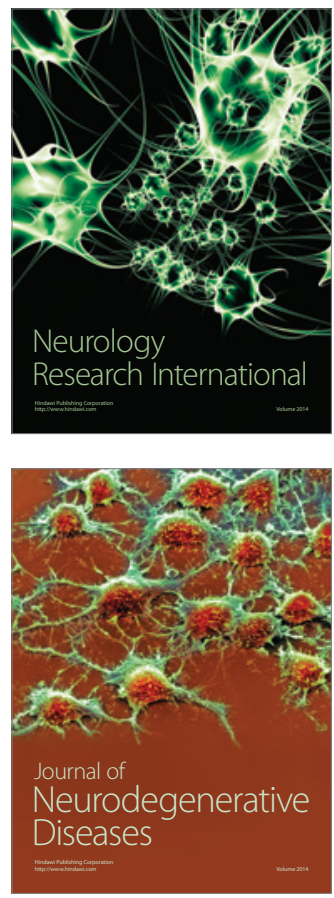

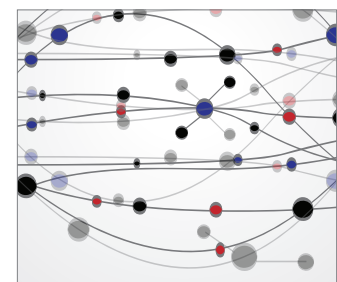

The Scientific World Journal
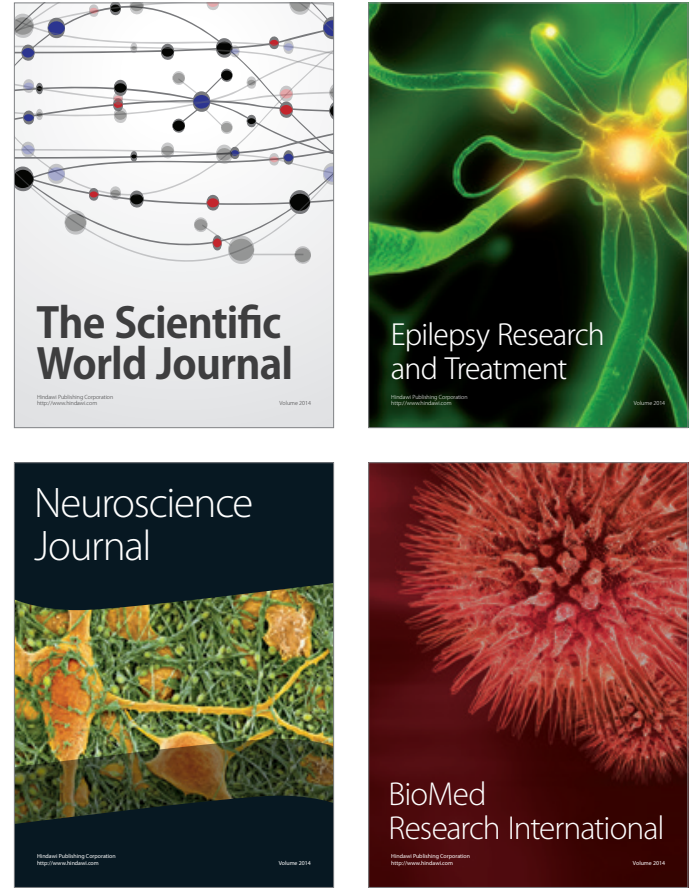

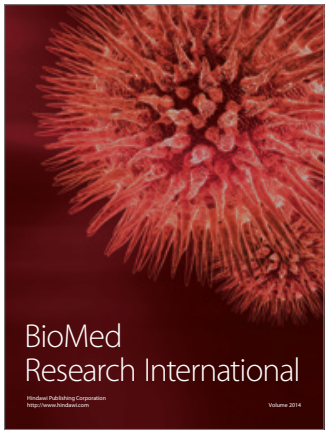

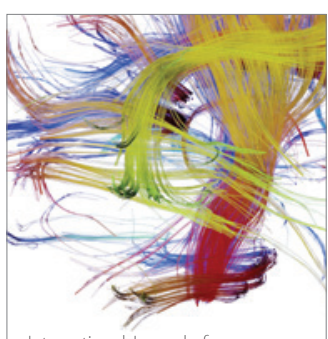

Brain Science

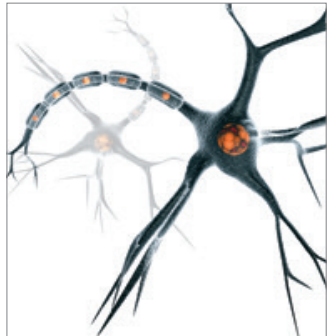

Neural Plasticity
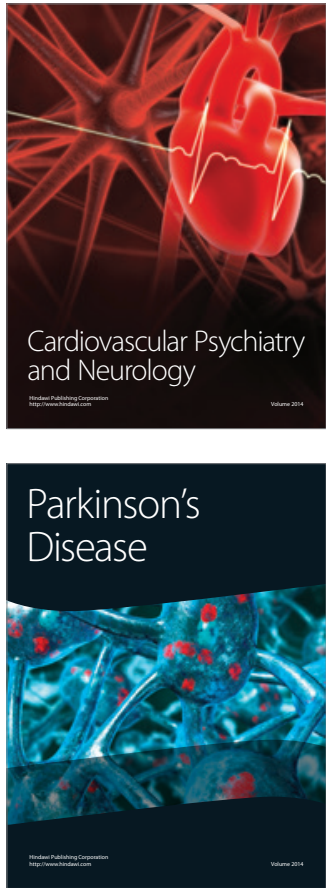